\title{
Research Article \\ Venturi Wet Gas Flow Modeling Based on Homogeneous and Separated Flow Theory
}

\author{
Fang Lide, ${ }^{1}$ Zhang Tao, $^{2}$ and Xu Ying ${ }^{2}$ \\ ${ }^{1}$ The Institute of Quality and Technology Supervising, Hebei University, Baoding 071051, China \\ ${ }^{2}$ School of Electrical and Automation Engineering, Tianjin University, Weijin Road no. 92, \\ Tianjin 300072, China
}

Correspondence should be addressed to Fang Lide, leed_amy@yahoo.com.cn

Received 16 April 2008; Accepted 27 May 2008

Recommended by Cristian Toma

When Venturi meters are used in wet gas, the measured differential pressure is higher than it would be in gas phases flowing alone. This phenomenon is called over-reading. Eight famous over-reading correlations have been studied by many researchers under low- and high-pressure conditions, the conclusion is separated flow model and homogeneous flow model performing well both under high and low pressures. In this study, a new metering method is presented based on homogeneous and separated flow theory; the acceleration pressure drop and the friction pressure drop of Venturi under two-phase flow conditions are considered in new correlation, and its validity is verified through experiment. For low pressure, a new test program has been implemented in Tianjin University's low-pressure wet gas loop. For high pressure, the National Engineering Laboratory offered their reports on the web, so the coefficients of the new proposed correlation are fitted with all independent data both under high and low pressures. Finally, the applicability and errors of new correlation are analyzed.

Copyright (C) 2008 Fang Lide et al. This is an open access article distributed under the Creative Commons Attribution License, which permits unrestricted use, distribution, and reproduction in any medium, provided the original work is properly cited.

\section{Introduction}

Wet gas metering has been described as a subset of multiphase flow measurement, where the volume of gas at actual measuring conditions is very high when compared to the volume of liquid in the flow stream. High-gas volume fraction has been defined in the range of $90-98 \%$ by different technical papers; more details are shown by Agar and Farchy [1]. Normally, these conditions need wet gas metering; for instance, some small or remote gas fields are processed together in common platform facilities, the individual unprocessed streams must be metered before mixing. In other circumstances, some gas meters may also be subjected to small amounts of liquid in the gas. This can happen to the gas output of a separator as a result of unexpected well conditions or liquid slugging. 
Table 1: Result of high-pressure comparison.

\begin{tabular}{lcc}
\hline Models & Root mean square error & Rank \\
\hline De Leeuw & 0.0211 & 1 \\
Homogeneous & 0.0237 & 2 \\
Lin & 0.0462 & 3 \\
Murdork 1.5 & 0.0482 & 4 \\
Murdork 1.26 & 0.0650 & 5 \\
Chisholm & 0.0710 & 6 \\
Smith and Leang & 0.1260 & 7 \\
\hline
\end{tabular}

Two ways are employed to meter wet gas: one approach is to use a multiphase flow meter in wet gas, and the other approach is to use a standard dry gas meter applying corrections to the measurements based on knowledge of how this type of meter is affected by the presence of liquid in the gas stream. This method requires prior knowledge of the liquid flow, which has to be obtained through another means; more details were shown by Lupeau et al. [2].

As a mature single-phase flow measurement device, the Venturi meter has been successfully applied in a variety of industrial fields and scientific research. Just owing to its successful applications in single-phase flows, the Venturi meter can easily be considered for two-phase flow measurement. When Venturi meters are used in wet gas, the measured differential pressure is higher than it would be with the gas phase flowing alone. If uncorrected, this additional pressure drop will result in an over reading of the gas mass flow rate. More details were shown by Geng et al. [3].

Eight famous over-reading correlations have been studied in low- and high-pressure conditions [4-10]. In Steven's paper [10], an ISA Controls standard North Sea specification 6 "Venturi meters with a 0.55 diameter ratio (or "beta") of $6 \mathrm{~mm}$ pressure tappings was the meter installed in National Engineering Laboratory (NEL) with pressure from 2 to $6 \mathrm{MPa}$ and LM parameter from 0 to 0.3 . NEL's engineer tested three 4 -inch meters with different beta values $(0.4,0.60,0.75)$ and tested over a range of pressures $(1.5-6.0 \mathrm{MPa})$ gas densimetric Froude number $\left(\mathrm{Fr}_{g}\right), 0.5-5.5$, and Lockhart-Martinelli parameter, $X, 0-0.4$ [11-13]. The results show that the liquid existence causes the meters to "over-read" the gas flow rate. This over reading is affected by the liquid fraction, gas velocity, pressure, and Venturi beta value. They predicted that some of the data seem to tend to a value slightly above unity, particularly at low $X$ values. Furthermore, in 2002, Britton et al. did some tests in Colorado Engineering Experiment Station, Inc., Colo, USA, [14, 15] with pressure between 1.4-7.6 MPa and $X$ values between $0-0.25$. Their study also confirmed the over-reading existence in Venturi meters.

The result of high-pressure comparison is shown in Table 1 [10].

Under low pressure, eight correlations are compared with Tianjin University's lowpressure wet gas test facilities [16] (see Table 2).

The method of comparing the seven correlations performances was chosen to be by comparison of the root mean square error (defined as $\delta$ ):

$$
\delta=\sqrt{\frac{1}{N} \sum_{1}^{N}\left(\frac{\mathrm{OR}_{p(i)}-\mathrm{OR}_{e(i)}}{\mathrm{OR}_{e(i)}}\right)^{2}}
$$


Table 2: Result of low-pressure comparison.

\begin{tabular}{lcc}
\hline Models & RMSE & Rank \\
\hline Homogenous & 0.11021 & 1 \\
Steven & 0.14787 & 2 \\
De Leeuw & 0.14854 & 3 \\
Smith and Leang & 0.18821 & 4 \\
Chisholm & 0.19597 & 5 \\
Murdock1.5 & 0.20658 & 6 \\
Lin & 0.20742 & 7 \\
Murdock & 0.21078 & 8 \\
\hline
\end{tabular}

where $\mathrm{OR}_{p(i)}$ is prediction over reading; $\mathrm{OR}_{e(i)}$ is experimentation over reading; $N$ is data numbers.

Tables 1 and 2 show the models performance in low and high pressure. By De Leeuw model being based on separated flow assumption, more parameters have been considered so it performs well. Although the assumptions of homogeneous models are simple, it performs well at both low pressure and high pressure (see Steven's results), for wet gas, homogeneous models may be true to some extent. This means that wet gas flow structure holds homogeneous character and separation character. Therefore, a new correlation considering homogeneous and separation flow theory together could be better than the previous ones.

This paper proposed a new Venturi wet gas correlation based on homogenous and separate assumption. The acceleration pressure drop and the friction pressure drop of Venturi under two-phase flow conditions are considered in new correlation, and its validity is verified through experiment. Finally, the performance of the new proposed correlations is compared with the old eight correlations both under low and high pressure.

\section{New Model Based on Homogeneous and Separated Flow Theory}

\subsection{Over-Reading Theory of Venturi Wet Gas Metering}

When Venturi meters are used in wet gas the measured differential pressure is higher than it would be for the gas phase flowing alone. If uncorrected, this additional pressure drop will result in an over reading of the gas mass flow rate:

$$
\mathrm{OR}=\frac{m_{g}^{\prime}}{m_{g}}
$$

where $m_{g}$ is the correct gas mass flow rate, $m_{g}^{\prime}$ is the apparent gas mass flow rate determined from the two-phase measured differential pressure $\Delta P_{\mathrm{tp}}, \Delta P_{\mathrm{tp}}$ is the actual 
two-phase differential pressure between the upstream and throat tappings, and $\Delta P_{g}$ is the gas differential pressure between the upstream and throat tappings:

$$
\begin{aligned}
& m_{g}=\frac{C \varepsilon A_{T} \sqrt{2 \rho_{g} \Delta P_{g}}}{\sqrt{1-\beta^{4}}}, \\
& m_{g}^{\prime}=\frac{C \varepsilon A_{T} \sqrt{2 \rho_{g} \Delta P_{\mathrm{tp}}}}{\sqrt{1-\beta^{4}}} .
\end{aligned}
$$

In (2.2) and (2.3), $C$ is discharge coefficient, $A_{T}$ is the area of the Venturi throat, $\varepsilon$ is expansibility factor, $\rho_{g}$ is gas density, and $\beta$ is diameter ratio. In fact, the discharge coefficient $C$ is variable under different flow conditions. Here, given that the discharge coefficient $C$ is constant, and take into account the fact that different flow conditions only have effect on over reading, but not have effect on the discharge coefficient given $C$.

The real gas mass flow rate can been obtained by

$$
m_{g}=\frac{m_{g}^{\prime}}{\mathrm{OR}}
$$

The homogeneous flow theory treats the two-phase flow as if it was a single-phase flow by using a homogeneous density expression $\rho_{\text {tp }}$ which averages the phase densities so that the single-phase differential pressure meter equation can be used

$$
\frac{1}{\rho_{\mathrm{tp}}}=\frac{x}{\rho_{g}}+\frac{1-x}{\rho_{l}}
$$

where $x$ is the mass quality, $\rho_{\text {tp }}$ is the homogeneous density, and subscripts " $l$ " and " $g$ " are for liquid and gas, respectively.

With this models the gas mass flow rate of the two phase flow can be written as

$$
m_{g}=x \frac{C \varepsilon A_{T} \sqrt{2 \rho_{\mathrm{tp}} \Delta P_{\mathrm{tp}}}}{\sqrt{1-\beta^{4}}} .
$$

Let (2.3) divide (2.6), then the homogeneous model gives

$$
\begin{aligned}
& \mathrm{OR}_{h}=\frac{m_{g}^{\prime}}{m_{g}}=\frac{C \varepsilon A_{T} \sqrt{2 \rho_{g} \Delta P_{\mathrm{tp}}} / \sqrt{1-\beta^{4}}}{x\left(C \varepsilon A_{T} \sqrt{2 \rho_{\mathrm{tp}} \Delta P_{\mathrm{tp}}} / \sqrt{1-\beta^{4}}\right)}, \\
& \mathrm{OR}_{h}=\frac{1}{x} \sqrt{\frac{\rho_{g}}{\rho_{l}}+\left(1-\frac{\rho_{g}}{\rho_{l}}\right) x .}
\end{aligned}
$$

However, (2.6) is also an estimation function about gas mass flow rate; the real gas mass flow rate should be (2.2) and then (2.6) as the apparent gas mass flow rate will be more 
rational. So let (2.6) divide (2.2), the real over reading under the homogeneous flow theory is shown in the following form:

$$
\begin{aligned}
\mathrm{OR}_{h} & =\frac{m_{g}^{\prime}}{m_{g}} \\
& =\frac{x \cdot\left(C \varepsilon A_{T} \sqrt{2 \rho_{\mathrm{tp}} \Delta P_{\mathrm{tp}}} / \sqrt{1-\beta^{4}}\right)}{C \varepsilon A_{T} \sqrt{2 \rho_{g} \Delta P_{g}} / \sqrt{1-\beta^{4}}} \\
& =x \cdot \sqrt{\frac{\rho_{\mathrm{tp}}}{\rho_{g}} \cdot \frac{\Delta P_{\mathrm{tp}}}{\Delta P_{g}}} .
\end{aligned}
$$

Equation (2.8) derived from homogeneous flow theory, if $\sqrt{\Delta P_{\mathrm{tp}} / \Delta P_{g}}$ derived from separation flow theory, the combination of homogeneous and separation flow theory is implemented.

Separated flow theory takes into account the fact that the two phases can have differing properties and different velocities. Separate equations of continuity, momentum, and energy are written for each phase, and these six equations are solved simultaneously, together with rate equations which describe how the phases interact with each other and with the walls of duct. In the simplest version, only one parameter, such as velocity, is allowed to differ for the two phases while conservation equations are only written for the combined flow.

Equation (2.9) shows the momentum function of one dimension two-phase flow based on separated flow assumption. The pressure drop of fluids in the pipe come from three parts, the first is friction; the second is gravitation; the third is acceleration [17-21]:

$$
\begin{aligned}
-\frac{d P}{d z}= & \frac{\tau_{0} U}{A}+\left[\rho_{g} \alpha+\rho_{1}(1-\alpha)\right] g \sin \theta \\
& +\frac{1}{A} \frac{d}{d z}\left\{A G^{2}\left[\frac{(1-x)^{2}}{\rho_{l}(1-\alpha)}+\frac{x^{2}}{\rho_{g} \alpha}\right]\right\}, \\
-\frac{d P}{d z}= & \frac{d P_{f}}{d z}+\frac{d P_{g}}{d z}+\frac{d P_{a}}{d z},
\end{aligned}
$$

where $\tau_{0}$ is friction force, $U$ is perimeter of pipe, $\alpha$ is void fraction, $G$ is mass velocity of mixture, $d P_{f} / d z$ is pressure drop caused by friction, $d P_{g} / d z$ is pressure drop caused by gravitation, $d P_{a} / d z$ is pressure drop caused by acceleration.

\subsection{The Friction Pressure Drop of Venturi Under Two-Phase Flow Condition}

For single-phase flow in straight pipe, the friction pressure drop can be calculated with

$$
\frac{d P_{f}}{d z}=\frac{\lambda}{d} \cdot \frac{\rho u^{2}}{2}
$$

where $\lambda$ is the friction factor; $d$ is the pipe diameter, $u$ is the velocity. 


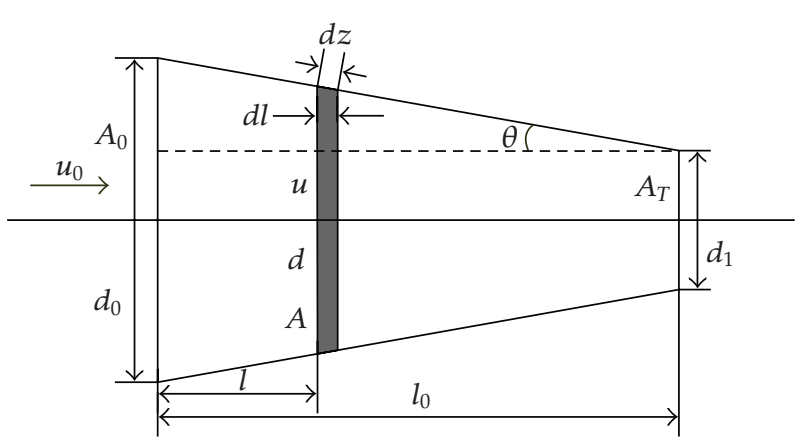

Figure 1: Sketch of Venturi conical convergent region.

Given $\lambda$ is constant in conical convergent of Venturi, the fluid velocity in the straight pipe keep unchanged, $d_{0}$ is diameter of straight pipe, $A_{0}$ is cross-section of straight pipe, $d_{1}$ is diameter of Venturi throat, $l_{0}$ is the length of conical convergent, $\theta$ is convergent angle. The schematic of Venturi conical convergent part is shown in Figure 1.

Analyzing an infinitesimal $d l$ given $d$ is diameter of the analyzing part, $A$ is crosssection, $l$ is the distance from Venturi inlet to infinitesimal $d l$ make integral to (2.11):

$$
\begin{aligned}
& \Delta P_{f}=\int_{0}^{l_{0}} \frac{\lambda}{d} \cdot \frac{\rho u^{2}}{2} d z, \\
& \Delta P_{f}=\frac{\lambda \rho}{2} \int_{0}^{l_{0}} \frac{1}{d} \cdot u^{2} d z .
\end{aligned}
$$

Multiply $d_{0}$ to (2.13) in two sides:

$$
\Delta P_{f}=\frac{\lambda \rho}{2 d_{0}} \int_{0}^{l_{0}} \frac{d_{0}}{d} \cdot u^{2} d z
$$

From continuity equation,

$$
\begin{gathered}
u=\frac{A_{0}}{A} u_{0}, \\
\frac{A_{0}}{A}=\left(\frac{d_{0}}{d}\right)^{2} .
\end{gathered}
$$

Substitute (2.15) into (2.14):

$$
\Delta P_{f}=\frac{\lambda \rho u_{0}^{2}}{2 d_{0}} \int_{0}^{l_{0}}\left(\frac{d_{0}}{d}\right)^{5} d z .
$$

According to geometrical relationship showed in Figure 1,

$$
\begin{gathered}
d z=\frac{d l}{\cos \theta}, \\
\frac{l}{l_{0}}=\frac{d_{0}-d}{d_{0}-d_{1}}, \\
\Longrightarrow \frac{d_{0}}{d}=\frac{l_{0}}{l_{0}-l\left(1-d_{1} / d_{0}\right)} .
\end{gathered}
$$


Let $\beta$ be diameter ratio of Venturi, then

$$
\beta=\frac{d_{1}}{d_{0}} .
$$

Substitute (2.17), (2.19), and (2.20) into (2.16):

$$
\begin{aligned}
& \Delta P_{f}=\frac{\lambda \rho u_{0}^{2} l_{0}^{5}}{2 d_{0} \cos \theta} \int_{0}^{l_{0}}\left(\frac{1}{l_{0}-l(1-\beta)}\right)^{5} d l, \\
& \Delta P_{f}=\left.\frac{\lambda \rho u_{0}^{2} l_{0}^{5}}{8 d_{0} \cos \theta(1-\beta)}\left(\frac{1}{l_{0}-l(1-\beta)}\right)^{4}\right|_{0} ^{l_{0}}, \\
& \Delta P_{f}=\frac{(1+\beta) \cdot\left(1+\beta^{2}\right)}{\beta^{4}} \cdot \frac{1}{4 \cos \theta} \cdot \frac{\lambda}{d_{0}} \cdot \frac{\rho u_{0}^{2}}{2} l_{0} .
\end{aligned}
$$

Equation (2.23a) shows that the friction pressure drop is affected by diameter ratio, convergent angle, convergent length, inlet diameter, and inlet velocity.

In a constant section pipe with $l_{0}$ length, the friction pressure drop is

$$
\Delta P_{f_{l 0}}=\frac{\lambda l_{0}}{d_{0}} \cdot \frac{\rho u_{0}^{2}}{2} \text {. }
$$

Equation (2.23a) that is divided by (2.23b) is

$$
K_{f}=\frac{(1+\beta) \cdot\left(1+\beta^{2}\right)}{\beta^{4}} \cdot \frac{1}{4 \cos \theta} .
$$

Equation (2.24) shows that the ratio $K_{f}$ is a function of diameter ratio and convergent angle. For a definite Venturi, $K_{f}$ is constant.

As for gas liquid two-phase flow, (2.23a) and (2.23b) changes into

$$
\Delta P_{f}=K_{f} \cdot \frac{\lambda l_{0}}{d_{0}} \cdot \frac{\alpha \rho_{g} u_{g}^{2}+(1-\alpha) \rho_{l} u_{l}^{2}}{2} .
$$

When the pipe is full of gas $(\alpha=1)$ or liquid $(\alpha=0),(2.25)$ changes to (2.23a).

From gas liquid two-phase flow continuity equation,

$$
\begin{aligned}
x G A & =A_{g} u_{g} \rho_{g}, \\
(1-x) G A & =A_{l} u_{l} \rho_{l} .
\end{aligned}
$$

Consider the definition of void fraction,

$$
\begin{gathered}
\frac{x}{\alpha} G=u_{g} \rho_{g}, \\
\frac{(1-x)}{(1-\alpha)} G=u_{l} \rho_{l}, \\
G=\frac{m}{A}=\alpha \rho_{g} u_{g}+(1-\alpha) \rho_{l} u_{l}
\end{gathered}
$$

which defined $S$ as slip ratio, that is, gas and liquid real velocity ratio combine (2.26) and (2.27):

$$
\frac{1}{\alpha}=1+s \frac{1-x}{x} \cdot \frac{\rho_{g}}{\rho_{l}}
$$


Substitute (2.26) and (2.27) into (2.25):

$$
\Delta P_{f}=K_{f} \cdot \frac{\lambda l_{0}}{d_{0}} \cdot \frac{G^{2}}{2} \cdot \frac{1}{\rho_{l}}\left[\frac{x^{2}}{\alpha} \cdot \frac{\rho_{l}}{\rho_{g}}+\frac{(1-x)^{2}}{1-\alpha}\right] .
$$

When the pipe is full of gas,

$$
\Delta P_{\mathrm{fg}}=K_{f} \cdot \frac{\lambda_{g} l_{0}}{d_{0}} \cdot \frac{G^{2}}{2} \cdot \frac{x^{2}}{\rho_{g}} .
$$

When the pipe is full of liquid,

$$
\Delta P_{\mathrm{fl}}=K_{f} \cdot \frac{\lambda_{l} l_{0}}{d_{0}} \cdot \frac{G^{2}}{2} \cdot \frac{(1-x)^{2}}{\rho_{l}} .
$$

Let $\lambda=\lambda_{g}=\lambda_{l}$, define $X_{f}$ as

$$
X_{f}=\sqrt{\frac{\Delta P_{f l}}{\Delta P_{f g}}}=\left(\frac{1-x}{x}\right) \sqrt{\frac{\rho_{g}}{\rho_{l}}} .
$$

Equation (2.30) divided by (2.31) is

$$
\frac{\Delta P_{f}}{\Delta P_{f g}}=\frac{1}{\alpha}+\frac{(1-x)^{2}}{x^{2}} \frac{\rho_{g}}{\rho_{l}} \cdot \frac{1}{1-\alpha}
$$

Substitute (2.29) into (2.34):

$$
\frac{\Delta P_{f}}{\Delta P_{f g}}=1+C_{f} X_{f}+X_{f}^{2}
$$

where

$$
C_{f}=\frac{1}{s} \sqrt{\frac{\rho_{l}}{\rho_{g}}}+s \sqrt{\frac{\rho_{g}}{\rho_{l}}} .
$$

\subsection{The Acceleration Pressure Drop of Venturi Under Two-Phase Flow Condition}

According to (2.9), the acceleration pressure drop is

$$
\begin{aligned}
& \frac{d P_{a}}{d z}=\frac{1}{A} \frac{d}{d z}\left\{A G^{2}\left[\frac{(1-x)^{2}}{\rho_{l}(1-\alpha)}+\frac{x^{2}}{\rho_{g} \alpha}\right]\right\} \\
& \Delta P_{a}=\int d P_{a}=\int_{A_{T}}^{A_{0}} \frac{1}{A} d\left\{A G^{2}\left[\frac{(1-x)^{2}}{\rho_{l}(1-\alpha)}+\frac{x^{2}}{\rho_{g} \alpha}\right]\right\} .
\end{aligned}
$$


Given the fluid is incompressible, the void fraction $\alpha$ is constant in the Venturi throat. Integrate (2.38):

$$
\Delta P_{a}=G^{2}\left[\frac{(1-x)^{2}}{\rho_{l}(1-\alpha)}+\frac{x^{2}}{\rho_{g} \alpha}\right] \cdot \ln \frac{A_{0}}{A_{T}} .
$$

When the gas was flowing alone in the pipe, the pressure drop can be expressed as

$$
\Delta P_{a g}=G^{2} \frac{x^{2}}{\rho_{g}} \cdot \ln \frac{A_{0}}{A_{T}} .
$$

The similar equation for the liquid phase is

$$
\Delta P_{a l}=\frac{G^{2}(1-x)^{2}}{\rho_{l}} \cdot \ln \frac{A_{0}}{A_{T}}
$$

Define $X_{a}$ :

$$
X_{a}=\sqrt{\frac{\Delta P_{a l}}{\Delta P_{a g}}}=\frac{(1-x)}{x} \cdot \sqrt{\frac{\rho_{g}}{\rho_{l}}} .
$$

Equation (2.39) divided by (2.40) is

$$
\frac{\Delta P_{a}}{\Delta P_{a g}}=\frac{(1-x)^{2}}{x^{2}} \cdot \frac{\rho_{g}}{\rho_{l}} \cdot \frac{1}{1-\alpha}+\frac{1}{\alpha} .
$$

Substitute (2.29) and (2.42) into (2.34):

$$
\frac{\Delta P_{a}}{\Delta P_{a g}}=1+C_{a} \cdot X_{a}+X_{a}^{2}
$$

where $C_{a}$ is expressed as

$$
C_{a}=\frac{1}{s} \cdot \sqrt{\frac{\rho_{l}}{\rho_{g}}}+s \cdot \sqrt{\frac{\rho_{g}}{\rho_{l}}} .
$$

Compare (2.33) with (2.42), it is obvious that $X_{f}$ is the same as $X_{a}$.

Also, compared (2.33) with (2.42), $C_{f}$ is equal to $C_{a}$.

And then, (2.44) is equal to

$$
\frac{\Delta P_{f}}{\Delta P_{f g}}=\frac{\Delta P_{a}}{\Delta P_{a g}}=1+C_{g} \cdot X+X^{2},
$$

where

$$
\begin{aligned}
& C_{g}=C_{a}=C_{f}=\frac{1}{s} \cdot \sqrt{\frac{\rho_{l}}{\rho_{g}}}+s \cdot \sqrt{\frac{\rho_{g}}{\rho_{l}}}, \\
& X=X_{a}=X_{f}=\frac{(1-x)}{x} \cdot \sqrt{\frac{\rho_{g}}{\rho_{l}}} .
\end{aligned}
$$

Equation (2.46) notes that the ratio of two-phase and single-phase friction pressure drop is equal to the ratio of two- phase and single-phase acceleration pressure drop. 


\subsection{The Total Pressure Drop of Venturi Under Two-Phase Flow Condition}

For a horizontal mounted Venturi, gravitation pressure drop can be ignored. The total pressure drop is

$$
\Delta P_{\mathrm{tp}}=\Delta P_{f}+\Delta P_{a} .
$$

The total pressure drop of Venturi under single-phase flow condition is

$$
\Delta P_{g}=\Delta P_{f g}+\Delta P_{a g}
$$

Divide (2.49) by (2.50):

$$
\frac{\Delta P_{\mathrm{tp}}}{\Delta P_{g}}=\frac{\Delta P_{f}+\Delta P_{a}}{\Delta P_{f g}+\Delta P_{a g}} .
$$

According to (2.46) and geometric axiom,

$$
\frac{\Delta P_{f}}{\Delta P_{f g}}=\frac{\Delta P_{a}}{\Delta P_{a g}}=\frac{\Delta P_{f}+\Delta P_{a}}{\Delta P_{f g}+\Delta P_{a g}} .
$$

Combine (2.51) and (2.52):

$$
\frac{\Delta P_{\mathrm{tp}}}{\Delta P_{g}}=\frac{\Delta P_{f}}{\Delta P_{f g}}=\frac{\Delta P_{a}}{\Delta P_{a g}}=1+C_{g} \cdot X+X^{2} .
$$

So the model combined homogeneous and separation flow theory can be expressed as (2.55). Call this correlation as $\mathrm{H}-\mathrm{S}$ model:

$$
\begin{aligned}
\mathrm{OR}_{\mathrm{H}-\mathrm{S}} & =x \cdot \sqrt{\frac{\rho_{\mathrm{tp}}}{\rho_{g}} \cdot \frac{\Delta P_{\mathrm{tp}}}{\Delta P_{g}}} \\
& =x \cdot \sqrt{\frac{\rho_{l}}{\rho_{l} x+\rho_{g}(1-x)}} \cdot \sqrt{1+C_{g} \cdot X+X^{2}} \\
& =\sqrt{x \cdot \frac{1+C_{g} \cdot X+X^{2}}{1+\sqrt{\rho_{g} / \rho_{l} X}}} .
\end{aligned}
$$

Equation (2.55) shows that $C_{g}$ is an effect factor to OR, it must be known first when (2.55) is used. However, slip ratio $S$ is contained in $C_{g}$ equation, and slip ratio is hard to be determined accurately, so it needs to fit a correlation with experiment.

\section{Dry Gas Calibration and Wet Gas Tests}

\subsection{Dry Gas Calibration}

Three venture meters are calibrated in TJU critical sonic nozzle flow calibration facility; see Figure 2. 


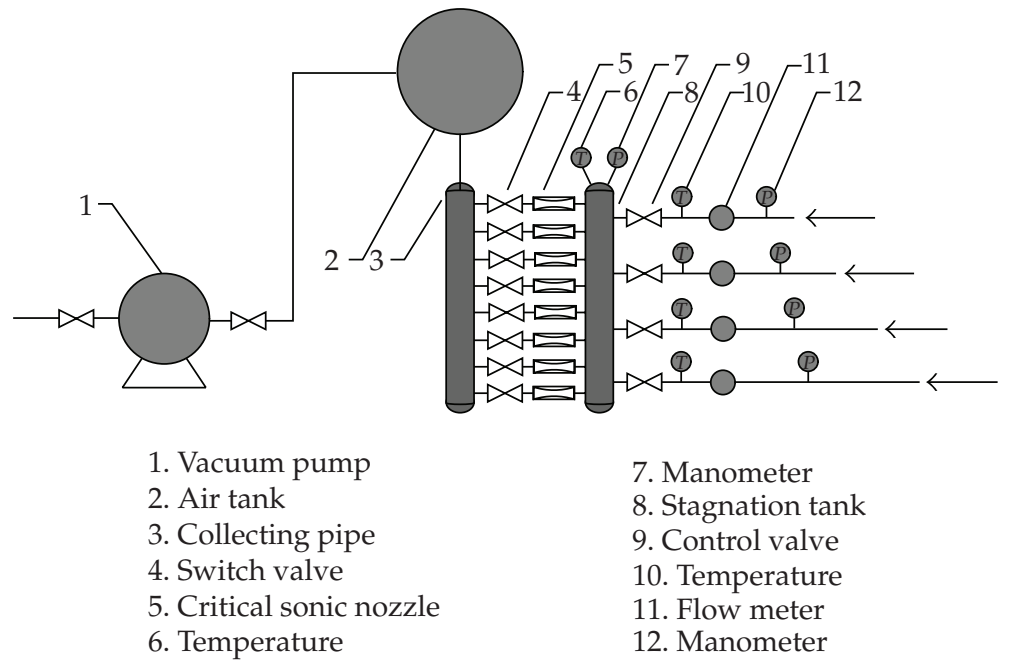

Figure 2: Schematic diagram of TJU critical sonic nozzle flow calibration facility.

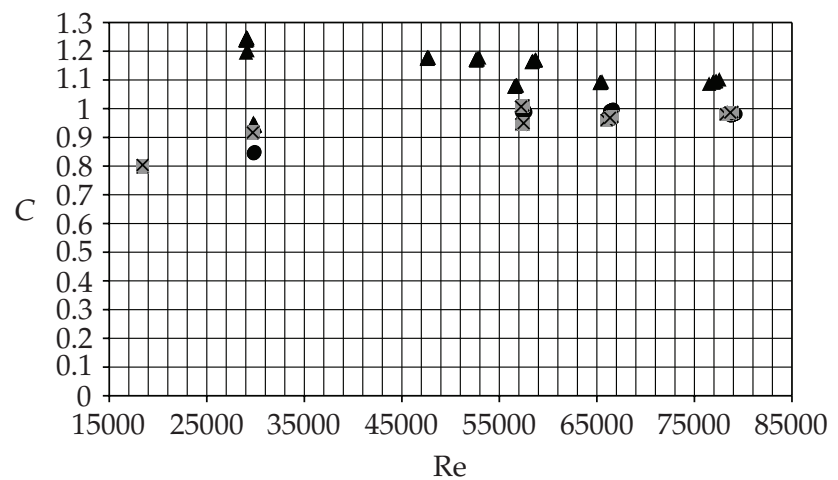

- 0.7

А 0.4

$\times 0.55$

Figure 3: Discharge coefficient of Venturi tube in single phase flow.

The facility has eleven sonic nozzles of different discharge coefficient, and the calibration range varies from 2.50 to $660 \mathrm{~m}^{3} / \mathrm{h}$ with a step of $2 \mathrm{~m}^{3} / \mathrm{h}$. The maximum calibrated flow rate is about $380 \mathrm{~m}^{3} / \mathrm{h}$ due to the beta ratio and pipe diameter. At the same time, the TJU multiphase flow loop also has the calibration function. So the dry gas calibration for three Venturis was done in both. The test data from the two facilities show the same results. Figure 3 shows the calibration coefficient $C$ with different diameter ratio. When the Reynolds number is higher than $1 \times 10^{5}$, the value of coefficient is in accord with the standard discharge coefficient for flows with Reynolds numbers less than one million [22].

Fit the coefficient $C$ in different diameter ratio, the parameters listed in table 3 :

$$
C=P_{1}+P_{2} \cdot \operatorname{Re}+P_{3} \cdot \operatorname{Re}^{2}+P_{4} \cdot \operatorname{Re}^{3}+P_{5} \cdot \operatorname{Re}^{4}+P_{6} \cdot \operatorname{Re}^{5}
$$




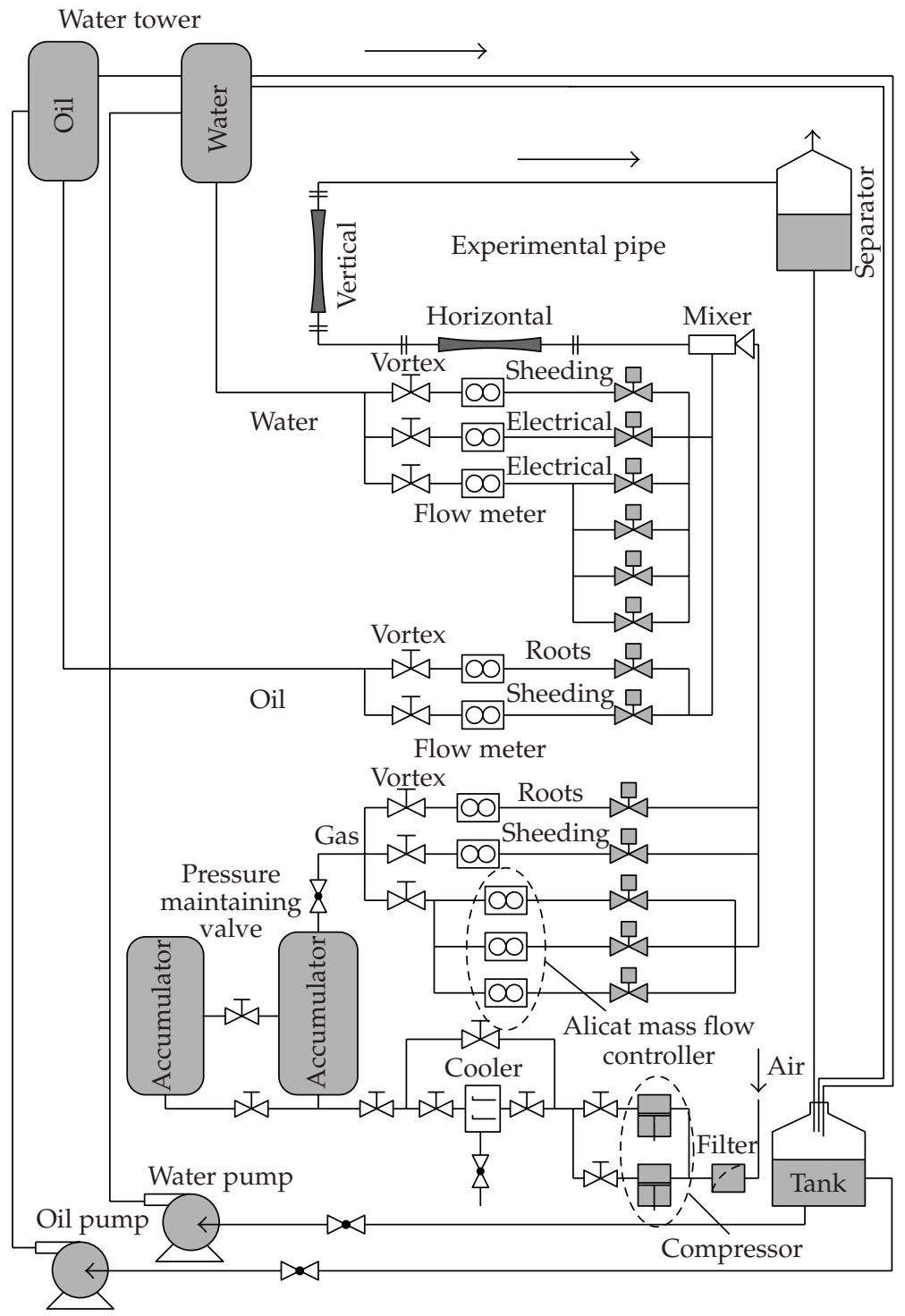

Figure 4: Schematic diagram of TJU multiphase flow loop.

\subsection{Test System and Experimental Procedures}

The tests were conducted on TJU multiphase flow loop at pressures from $0.15 \mathrm{MPa}$ to $0.25 \mathrm{MPa}$ across a range of gas velocities and liquid fractions. TJU's low-pressure wet gas test facilities are a fully automatic control and functional complete system, which is not only a multiphase flow experiment system, but also a multiphase flow meter calibration system. As an experiment system, the test can be conducted in a horizontal pipe, vertical pipe and $0-90^{\circ}$ lean pipe; as a calibration system, the test meter can be calibrated in standard meter method. Figure 4 shows schematic diagram of TJU multiphase flow loop.

Thess facilities have six components, named as medium source, measurement pipe, horizontal pipe, vertical pipe, $0-90^{\circ}$ lean pipe and computer control system. 


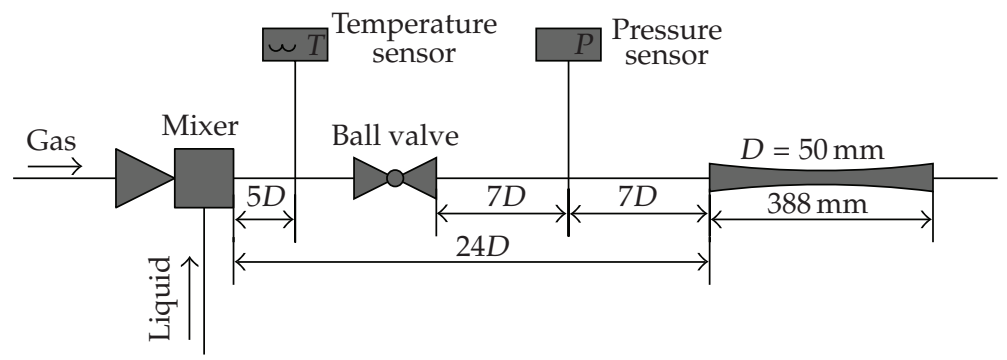

Figure 5: Horizontal experiment pipe.

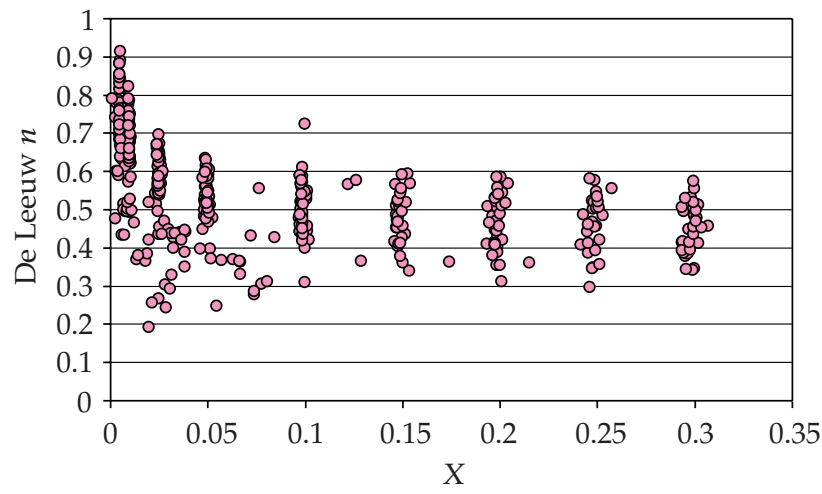

Figure 6: Lockhart-Martinelli parameter X effect on $n$ of De Leeuw model.

Gas medium is compression air, and two compressors provide dynamic force, the compressor air is passing through cooling and drying unit which access to two $12 \mathrm{~m}^{3}$ accumulator tanks; the accumulator tanks and pressure maintaining valve can hold a stable pressure $0-0.8 \mathrm{MPa}$ for the test. The liquids used in test are water (oil or oil and water mixture also can be used) and a water pump pushes the water to a 30-meter-high water tower, which can hold a stable pressure for liquid.

In standard meter calibration system, gas calibration system has five paths; three of them are low-flow channels metering with three mass flow controllers made in America by Alicat scientific company, Ariz, USA, the lowest flux is $101 / \mathrm{min}$, the other two paths are middle and large flow channels metering with a Roots type flow meter and a vortex flow meter. All temperature and pressure measurements use traceable calibrated instrumentation for gas temperature and pressure compensation.

Liquid calibration system has six paths: four of them are low-flow channels metering with an electrical flow meter made in Germany combined by four magnet valves, the lowest flux is $0.01 \mathrm{~m}^{3} / \mathrm{h}$, the other two paths are middle and large flow channels metering with a electrical flow meter and a vortex flow meter. See parameters of the standard meter in Table 4.

Gas and liquid calibrate through standard meter access to mixer, and then go through the experimental pipe. There are two paths in experimental pipes, one is made in rustless steel, the other is made in organic glass, their diameter is $50 \mathrm{~mm}$, and a cutoff valve which can adjust the pressure is installed at outlet of the pipe.

Figure 5 shows horizontal experiment pipe, which includes mixer, temperature sensor, straight lengths, pressure sensor, and Venturi tube. 
Table 3: Parameters value.

\begin{tabular}{lccc}
\hline & 0.4048 & 0.55 & 0.7 \\
\hline$P_{1}$ & 17.88251 & -0.58867 & 6.06974 \\
$P_{2}$ & -0.00074 & 0.00006 & -0.00016 \\
$P_{3}$ & $1.283 E-8$ & $-9.7022 E-10$ & $1.88 E-9$ \\
$P_{4}$ & $-1.0944 E-13$ & $8.4168 E-15$ & $-1.0733 E-14$ \\
$P_{5}$ & $4.5672 E-19$ & $-3.5094 E-20$ & $2.9549 E-20$ \\
$P_{6}$ & $-7.4476 E-25$ & $5.5411 E-26$ & $-3.1419 E-26$ \\
\hline
\end{tabular}

Table 4: Parameters of the standard sensor.

\begin{tabular}{lcc}
\hline Phases & Range $\left(\mathrm{m}^{3} / \mathrm{h}\right)$ & Accuracy \\
\hline \multirow{2}{*}{ Water } & $0.01 \sim 3.0$ & $\pm 0.2 \%$ \\
& $0.75 \sim 19$ & $\pm 1.0 \%$ \\
& $1.7 \sim 43$ & $\pm 0.5 \%$ \\
\multirow{2}{*}{ Air } & $0 \sim 6.0$ & $\pm 0.8 \%$ \\
& $0.15 \sim 17$ & $\pm 3.0 \%$ \\
& $6.5 \sim 130$ & $\pm 1.5 \%$ \\
Oil & $0.02 \sim 2.5$ & $1.0 \%$ \\
& $0.75 \sim 19$ & $1.0 \%$ \\
\hline
\end{tabular}

Table 5: Required straight lengths for classical Venturi tubes with a machined convergent section.

\begin{tabular}{lc}
\hline Diameter ratio & Straight length $(D)$ \\
\hline 0.40 & 8 \\
0.50 & 8 \\
0.60 & 10 \\
0.70 & 10 \\
0.75 & 18 \\
\hline
\end{tabular}

According to ISO 5167-1, 4 : 2003 [23, 24], a classical Venturi tube with a machined convergent section, straight lengths and diameter ratio must accord with Table 5.

In this test, three Venturi tubes with $\beta$ values of $0.4048,0.55$, and 0.70 have been produced, the length of Venturi tubes is $388 \mathrm{~mm}$, diameter is $50 \mathrm{~mm}$, the length of cylindrical throat is $20 \mathrm{~mm}$, conical convergent angle is $21^{\circ}$, conical divergent angle is $15^{\circ}$, diameter of pressure tappings is $4 \mathrm{~mm}$, the pipe wall roughness is $0.06 \mathrm{~mm}$, and stainless steel flange is used in connecting. 1151 differential pressure transducers were made in Rosemont company, Colo, USA, the uncertainty of whole equipment is $2.5 \%$.

The test data are collected and saved as Microsoft Excel file automatically (see experimental parameters in Table 6).

The flow pattern of the test included annular and drop-annular, where $\mathrm{Fr}_{g}$ is gas Froude number:

$$
\operatorname{Fr}_{g}=\frac{v_{g}}{\sqrt{g D}} \sqrt{\frac{\rho_{g}}{\rho_{l}-\rho_{g}}} .
$$

$v_{g}$ is superficial velocity of the gas phase: $v_{g}=m_{g} /\left(\rho_{g} A\right)$. 
Table 6: Experimental parameters.

\begin{tabular}{lccc}
\hline$\beta$ & $P(\mathrm{MPa})$ & $\mathrm{Fr}_{g}$ & $X$ \\
\hline \multirow{3}{*}{0.4048} & 0.15 & $0.8 \sim 1.5$ & $0.0022 \sim 0.0338$ \\
& 0.20 & $1.0 \sim 1.88$ & $0.0022 \sim 0.0472$ \\
& 0.25 & $0.67 \sim 1.81$ & $0.0022 \sim 0.0495$ \\
0.55 & 0.15 & $1.04 \sim 1.78$ & $0.0022 \sim 0.2572$ \\
& 0.20 & $1.09 \sim 1.85$ & $0.0022 \sim 0.3431$ \\
& 0.25 & $0.92 \sim 1.73$ & $0.0022 \sim 0.3514$ \\
0.7 & 0.15 & $1.04 \sim 2.0$ & $0.0024 \sim 0.0480$ \\
& 0.20 & $1.08 \sim 2.0$ & $0.0025 \sim 0.0525$ \\
& 0.25 & $0.87 \sim 1.66$ & $0.0027 \sim 0.0576$ \\
\hline
\end{tabular}

Table 7: Fit exponent $n$ with all data.

\begin{tabular}{ccccccccc}
\hline$a_{1}$ & $a_{2}$ & $a_{3}$ & $a_{4}$ & $a_{5}$ & $a_{6}$ & $a_{7}$ & $a_{8}$ & $K$ \\
\hline 1.29203 & -0.17161 & 0.12618 & -0.01884 & 0.30196 & 0.05205 & -0.07122 & 0.0259 & 0.78105 \\
\hline
\end{tabular}

\section{Model Parameters Determining and Error Analyzing}

The coefficient $C_{g}$ can be calculated by experimental data. On TJU multiphase flow system, the real gas, liquid mass flow rateand gas, liquid density can be determined by standard sensor. The gas mass fraction is known parameter. The Lockhart-Martinelli parameter can be obtained by (2.33). The over reading can be calculated with (2.1) and (2.3). Therefore, the coefficient $C_{g}$ can be calculated by (2.55) (H-S model). The study shows that coefficient $C_{g}$ decreases with increasing Lockhart-Martinelli parameter $X$, decreases with increasing pressure $P$, decreases with increasing diameter ratio $\beta$, increases with increasing Gas Froude number $\mathrm{Fr}_{g}$, and increases with increasing gas liquid quality ratio $x /(1-x)$.

Equation (2.47) can be expressed as

$$
C_{g}=f\left(s, \sqrt{\frac{\rho_{g}}{\rho_{l}}}\right) .
$$

Equation (4.1) shows that the gas-liquid quality ratio $x /(1-x)$ contains the same parameter with coefficient $C_{g}$ :

$$
\frac{m_{g}}{m_{l}}=\frac{x}{1-x}=S \cdot \frac{\rho_{g}}{\rho_{l}} \cdot \frac{\alpha}{1-\alpha} .
$$

Combining (3.2) and (4.1) can gain

$$
C_{g}=f\left(\frac{x}{1-x}, \frac{\alpha}{1-\alpha}, \sqrt{\frac{\rho_{g}}{\rho_{l}}}\right) .
$$

De Leeuw model considers the coefficient $C_{g}$ as a function of gas-liquid density ratio and gas Froude number:

$$
C_{\text {De Leeuw }}=\left(\frac{\rho_{l}}{\rho_{g}}\right)^{n}+\left(\frac{\rho_{g}}{\rho_{l}}\right)^{n}, \quad n= \begin{cases}0.41 & 0.5 \leq \mathrm{Fr}_{g} \leq 1.5 \\ 0.606\left(1-e^{-0.746 \mathrm{Fr}_{g}}\right) & \mathrm{Fr}_{g} \geq 1.5\end{cases}
$$




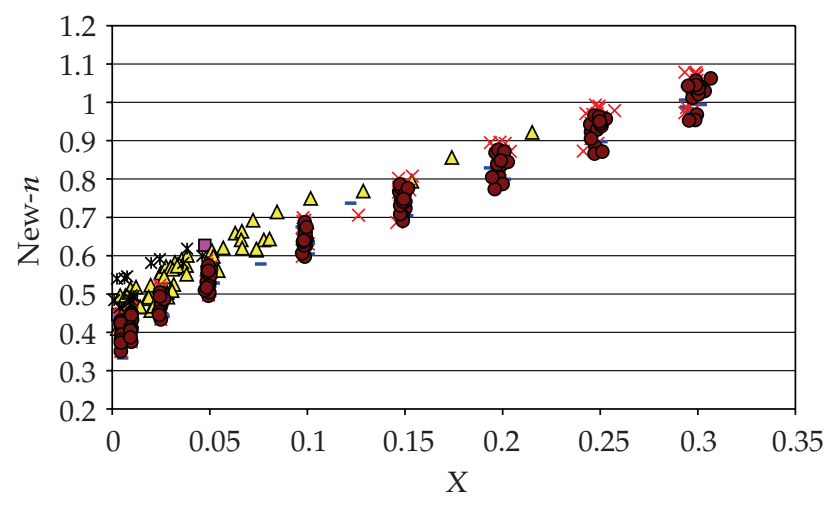
$-0.4$
$\times 0.6$
- 0.4048
* 0.7
$\triangle 0.55$
- 0.75

Figure 7: Lockhart-Martinelli parameter X effect to $n$.

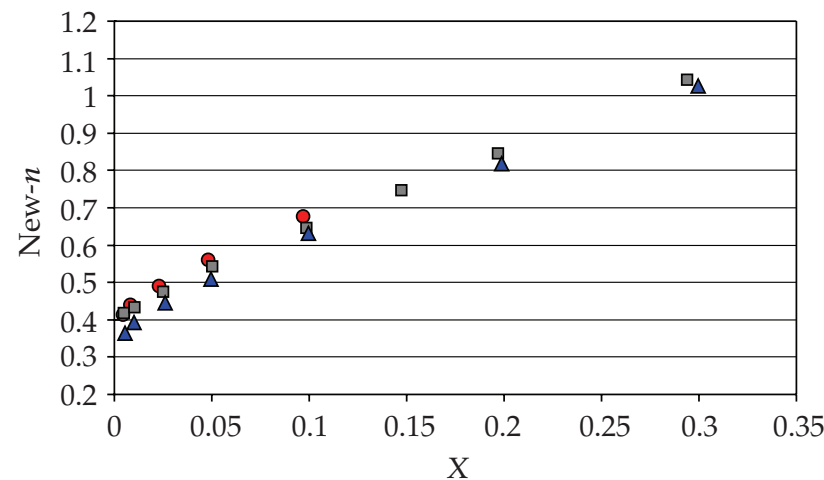

- $0.4, P=1.5 \mathrm{MP}$

- $0.4, P=3 \mathrm{MP}$

$\Delta 0.4, P=6 \mathrm{MP}$

Figure 8: Pressure $P$ effect on coefficient $n$ under same gas Froude number.

However, inherited the form of the coefficient $C_{g}$ of De Leeuw model's, and using gas liquid density ratio as base of exponential function, the exponent $n$ is a severe nonlinear curve with other parameters such as Lockhart-Martinelli parameter $X$, or gas Froude number (see Figure 6).

Research found that using gas liquid volume ratio (gas liquid mass ratio divided by gas liquid density ratio) as a base of exponential function $C_{g}$ in H-S model, the exponent $n$ almost linear increases with increasing Lockhart-Martinelli parameter $X$, it can be seen as Figure 7, so defined the coefficient $C_{g}$ of the H-S model as

$$
C_{\mathrm{H}-\mathrm{S}}=\left(\frac{x /(1-x)}{\rho_{g} / \rho_{l}}\right)^{n}+\left(\frac{\rho_{g} / \rho_{l}}{x /(1-x)}\right)^{n} .
$$




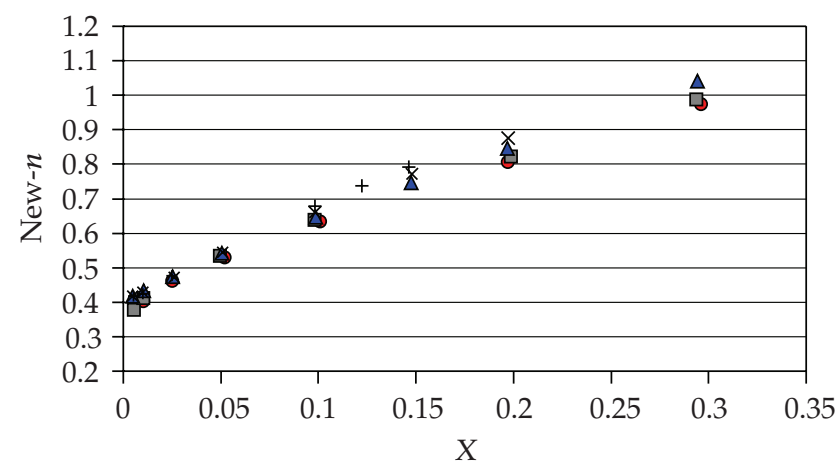
- $0.4, F r_{g}=1$
ㅁ $0.4, F r_{g}=1.5$
$\times 0.4, F r_{g}=2.5$
$+0.4, F r_{g}=3$
$\Delta 0.4, F r_{g}=2$

Figure 9: Gas Froude number $\mathrm{Fr}_{g}$ effect on coefficient $n$ under same pressure.

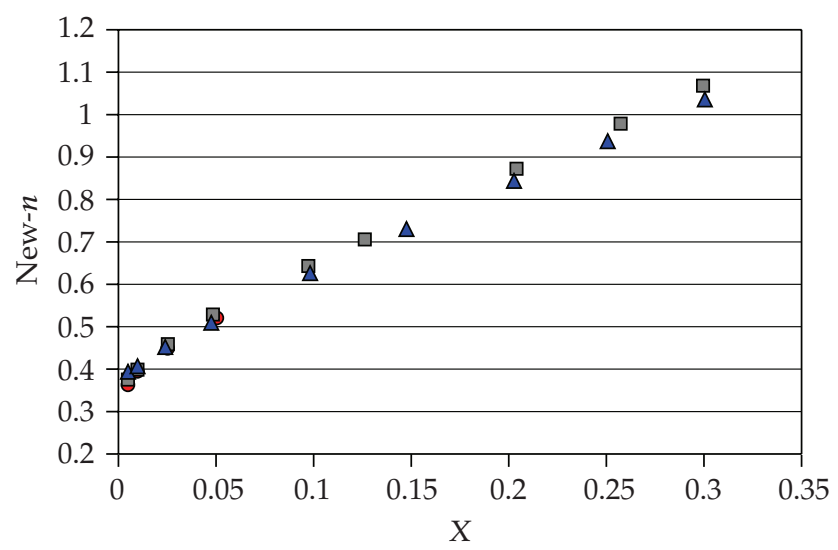

$$
\begin{array}{ll}
- & 0.4, F r_{g}=3.5 \\
\square & 0.6, F r_{g}=3.5 \\
\Delta & 0.75, F r_{g}=3.5
\end{array}
$$

Figure 10: Diameter ratio effect on coefficient $n$ under $6 \mathrm{MPa}$.

In fact, gas liquid mass ratio divided by gas liquid density ratio is equal to gas liquid volume ratio:

$$
\begin{aligned}
C_{\mathrm{H}-\mathrm{S}} & =\left(\frac{\varphi}{1-\varphi}\right)^{n}+\left(\frac{1-\varphi}{\varphi}\right)^{n}, \\
n & =f\left(\beta, P\left(\mathrm{OR} \frac{\rho_{g}}{\rho_{l}}\right), \mathrm{Fr}_{g}, X, \ldots\right),
\end{aligned}
$$

where $\varphi$ is gas volume fraction.

Next, a correlation of exponent $n$ with other parameters will be approached. 


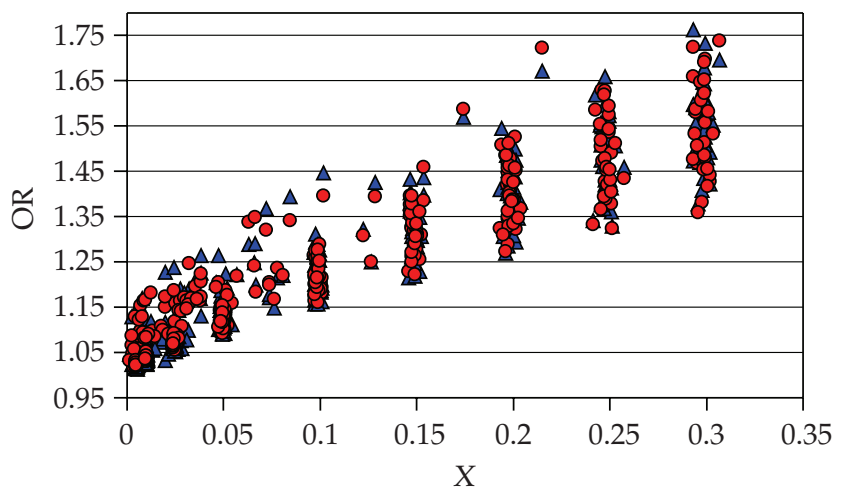

$\Delta$ Real OR

- OR-new-fit

Figure 11: Comparison of H-S prediction OR and experimental OR.

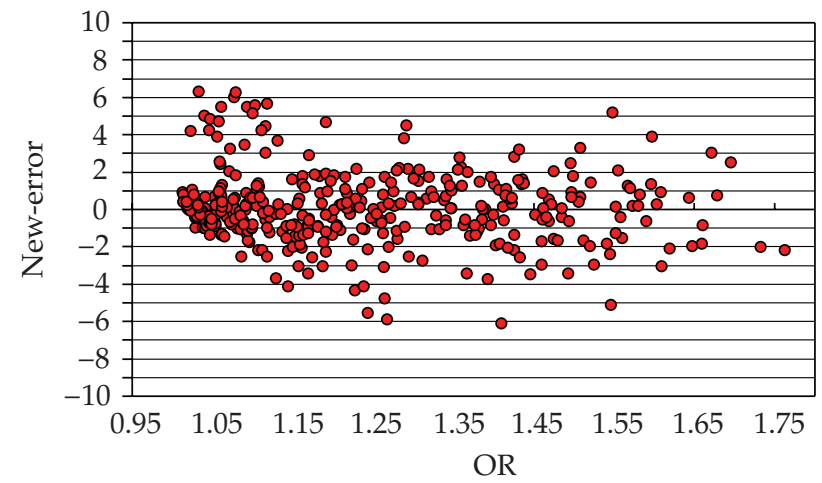

Figure 12: The prediction error of H-S model.

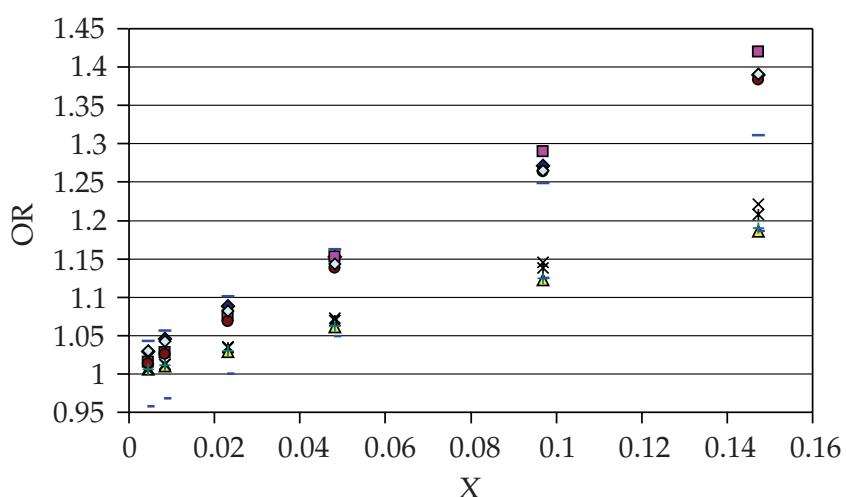

- Real OR

- Homogenous

$\Delta$ Murdock

- De Leeuw

$\times$ Murdock1

+ Lin Zh

- Smith and Leang

* Chisholm

- Steven

$\diamond \mathrm{H}-\mathrm{S}$

Figure 13: Comparison of models under $\beta=0.4, P=1.5 \mathrm{MPa}$, and $\mathrm{Fr}_{g}=2$. 


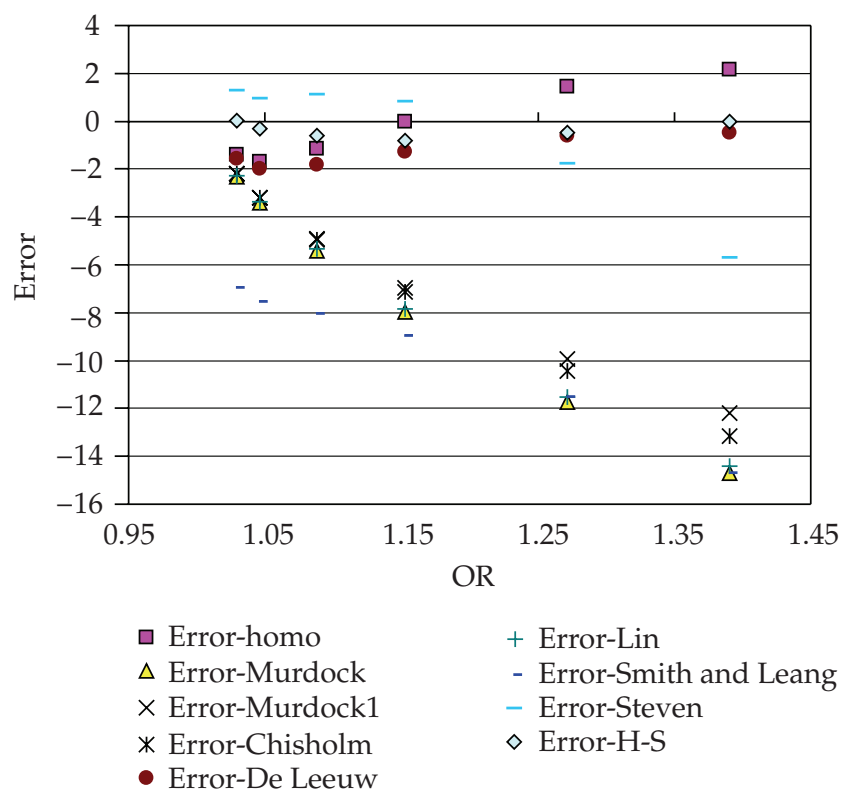

Figure 14: Comparison errors of models under $\beta=0.4, P=1.5 \mathrm{MPa}$, and $\mathrm{Fr}_{g}=2$.

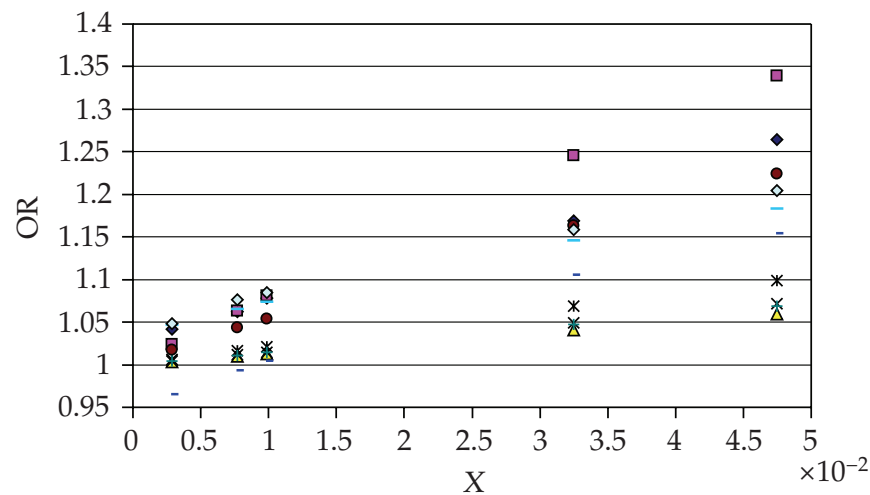
- Real OR
- Homogenous
- De Leeuw
$\triangle$ Murdock
+ Lin Zh
$\times$ Murdock1
- Smith and Leang
* Chisholm
- Steven
$\diamond \mathrm{H}-\mathrm{S}$

Figure 15: Comparison of models under $\beta=0.4048, P=0.20 \mathrm{MPa}$, and $\mathrm{Fr}_{g}=1.5$.

\subsection{Effect of Parameters to Exponent $n$ of $H-S$ Model}

Figure 7 shows the effect of Lockhart-Martinelli parameter $X$ to $n$, and exponent $n$ almost linearly increases with increasing Lockhart-Martinelli parameter $X$. Figure 8 shows the effect of pressure to $n$, apparently, exponent $n$ decreases with the increasing pressure. Figure 9 shows the effect of gas Froude number to $n$, seemingly, $n$ increases with the increasing of gas Froude number. Figure 10 shows the effect of diameter ratio to $n$, and $n$ decreases with the increasing diameter ratio. 


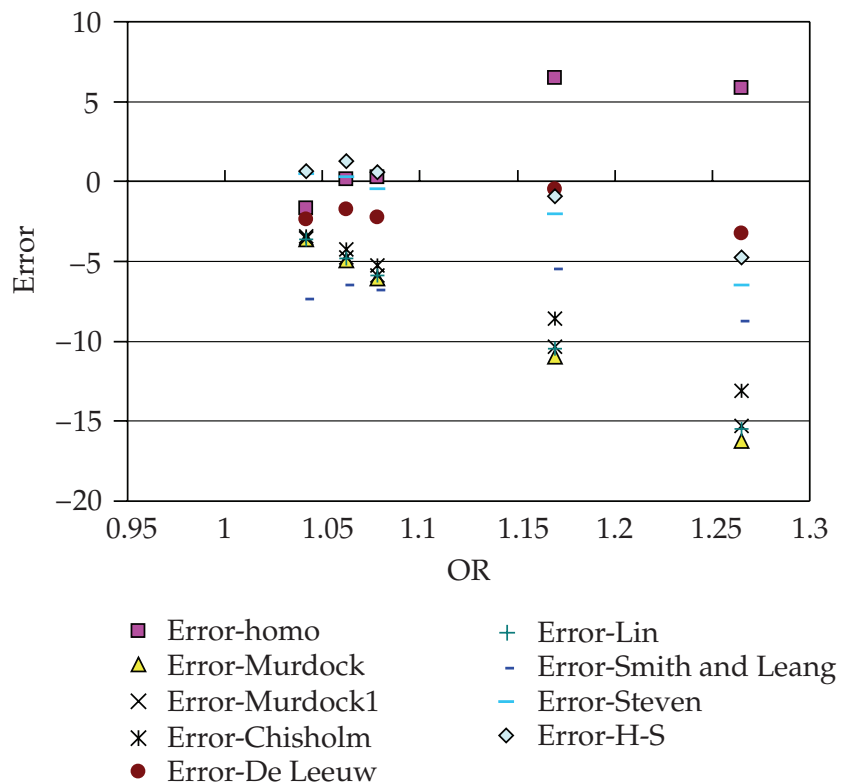

Figure 16: Comparison errors of models under $\beta=0.4048, P=0.20 \mathrm{MPa}$, and $\mathrm{Fr}_{g}=1.5$.

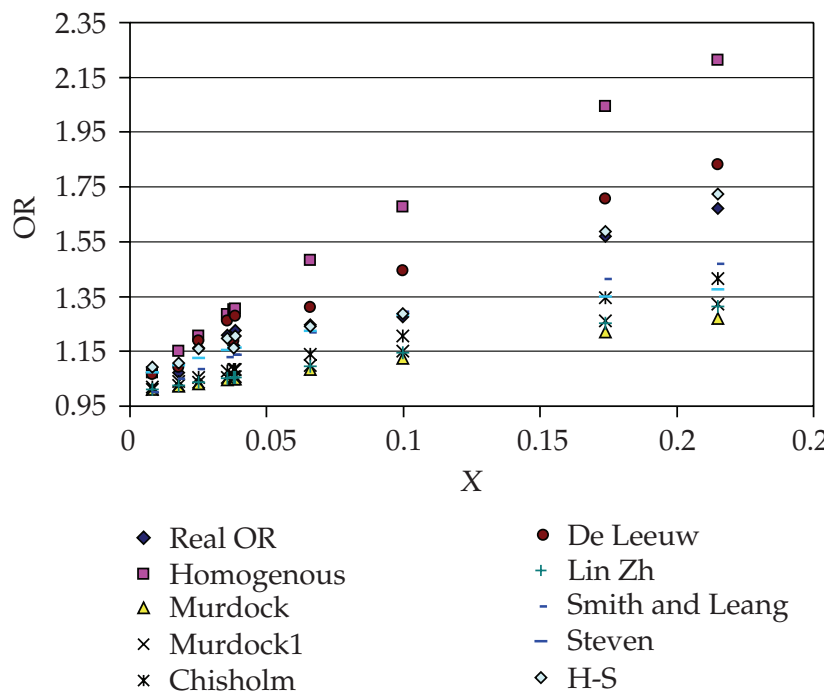

Figure 17: Comparison of models under $\beta=0.55, P=0.15 \mathrm{MPa}$, and $\mathrm{Fr}_{g}=2$.

\subsection{Fitting Exponent $n$ of H-S Model}

According to the results of these figures, $n$ varied linearly with Lockhart-Martinelli parameter $X$, and with the rate of curves effect by diameter ratio, pressure, and Gas Froude number. So the experiment correlation of coefficient $n$ should take the Lockhart-Martinelli parameter $X$ as a key independent variable, and pressure $P$ (or gas liquid density ratio), diameter ratio $\beta$, 


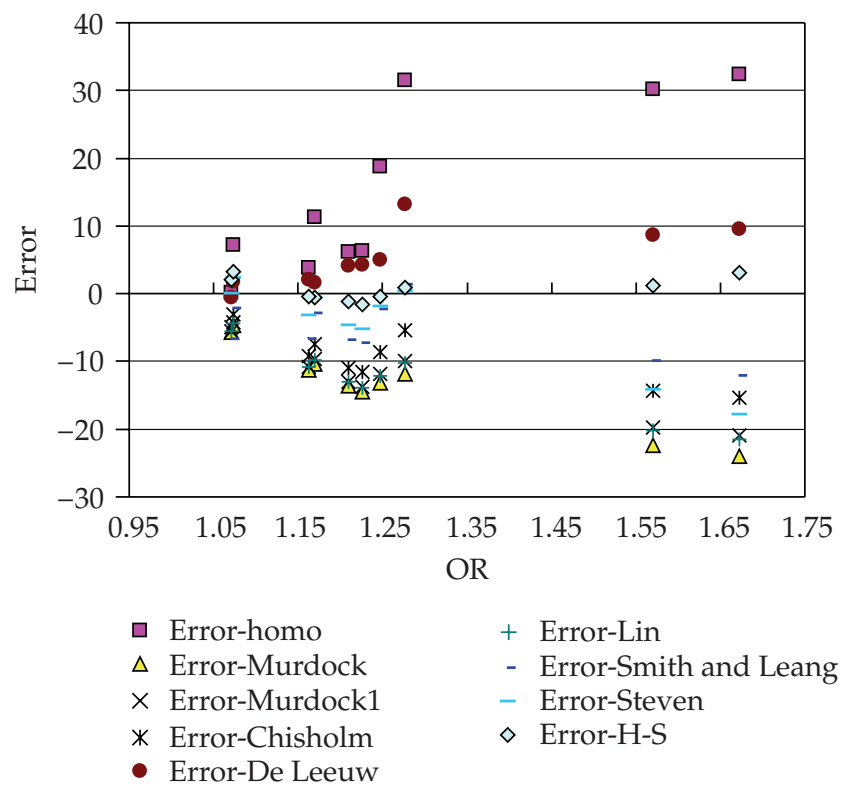

Figure 18: Comparison errors of models under $\beta=0.55, P=0.15 \mathrm{MPa}$, and $\mathrm{Fr}_{g}=2$.

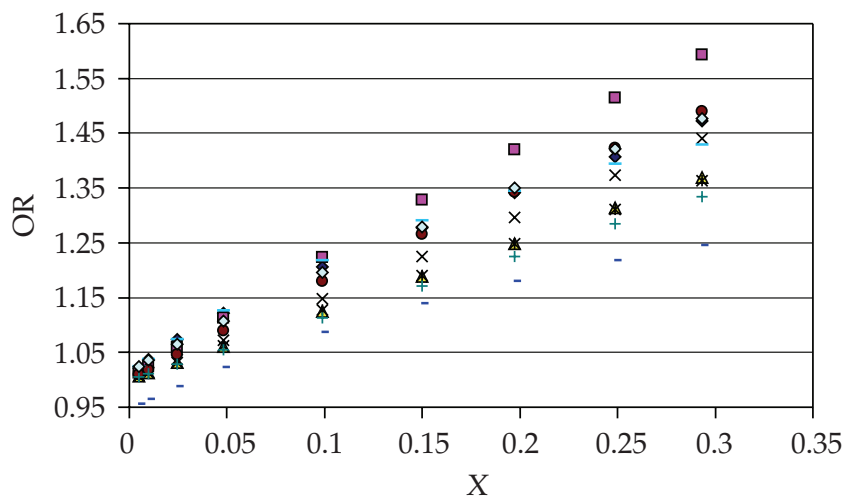
- Real OR
- Homogenous
- De Leeuw
$\triangle$ Murdock
+ Lin Zh
$\times$ Murdock1
- Smith and Leang
* Chisholm
- Steven
$\diamond \mathrm{H}-\mathrm{S}$

Figure 19: Comparison of models under $\beta=0.60, P=3 \mathrm{MPa}$, and $\mathrm{Fr}_{g}=1.5$.

Gas Froude number $\mathrm{Fr}_{g}$ as auxiliary variable. Exponent $n$ can be defined as

$$
n=A+B \cdot X^{k},
$$

where

$$
\begin{aligned}
& A=a_{1} \cdot(\beta)^{a_{2}} \cdot\left(\operatorname{Fr}_{g}\right)^{a_{3}} \cdot\left(\frac{\rho_{g}}{\rho_{l}}\right)^{a_{4}}, \\
& B=a_{5} \cdot(\beta)^{a_{6}} \cdot\left(\operatorname{Fr}_{g}\right)^{a_{7}} \cdot\left(\frac{\rho_{g}}{\rho_{l}}\right)^{a_{8}},
\end{aligned}
$$




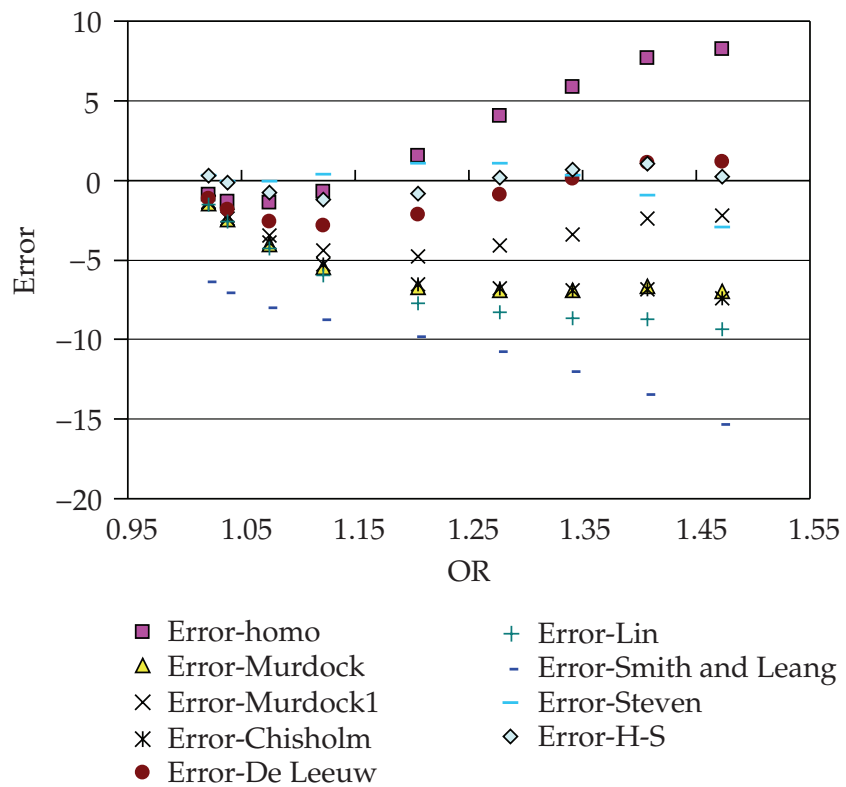

Figure 20: Comparison errors of models under $\beta=0.60, P=3 \mathrm{MPa}$, and $\mathrm{Fr}_{g}=1.5$.

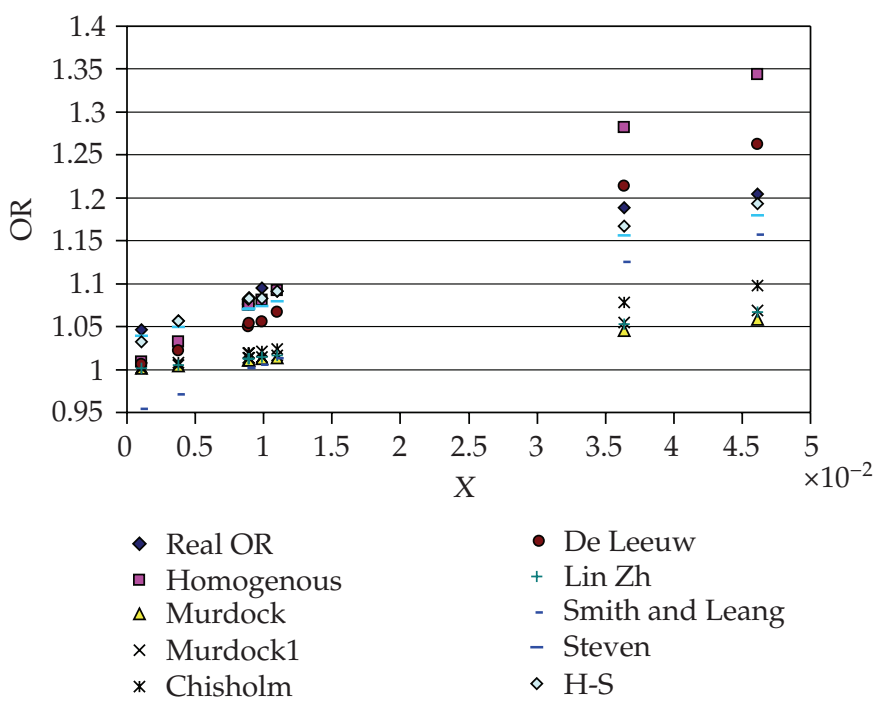

Figure 21: Comparison of models under $\beta=0.70, P=0.20 \mathrm{MPa}$, and $\mathrm{Fr}_{g}=1.7$.

where $K$ is constant, $a_{1}, a_{2}, a_{3}, a_{4}, a_{5}, a_{6}, a_{7}, a_{8}$ are undetermined coefficient, which will be determined through experimental data. The fit coefficient showed in Table 7.

Table 7 is the coefficient $n$ fit by independent data from TJU low-pressure wet gas loop and National Engineering Laboratory high-pressure wet gas loop. Using exponent $n$ and coefficient $C_{g}$ for $\mathrm{H}-\mathrm{S}$ over-reading model, over $98 \%$ data set will express the prediction error within $\pm 5 \%$, and the maximum error within $\pm 6.5 \%$. See Figures 11 and 12 . 


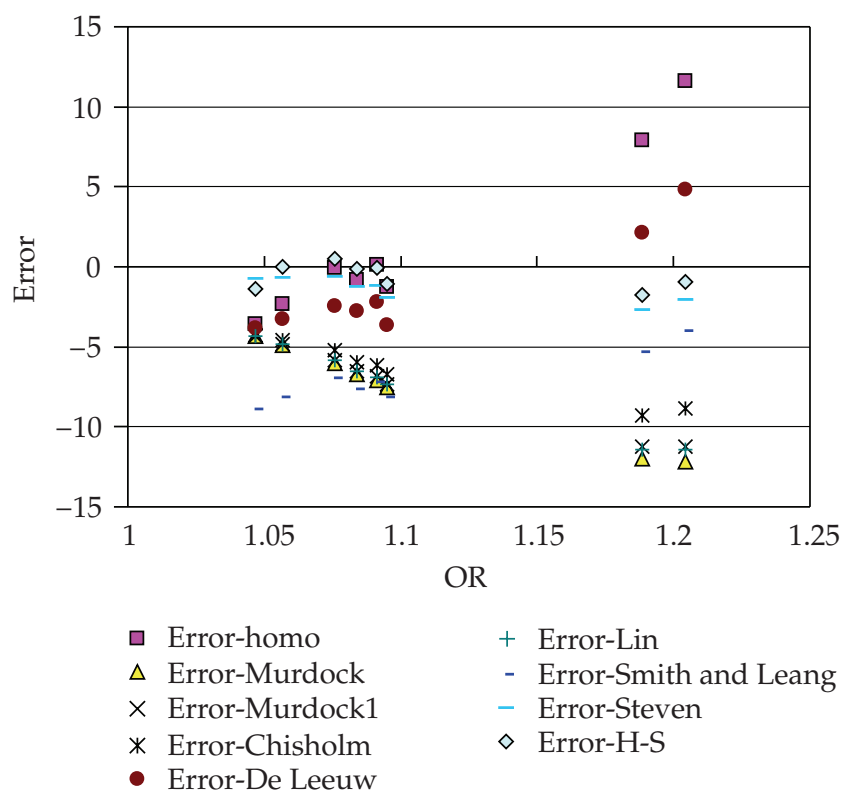

Figure 22: Comparison errors of models under $\beta=0.70, P=0.20 \mathrm{MPa}$, and $\mathrm{Fr}_{g}=1.7$.

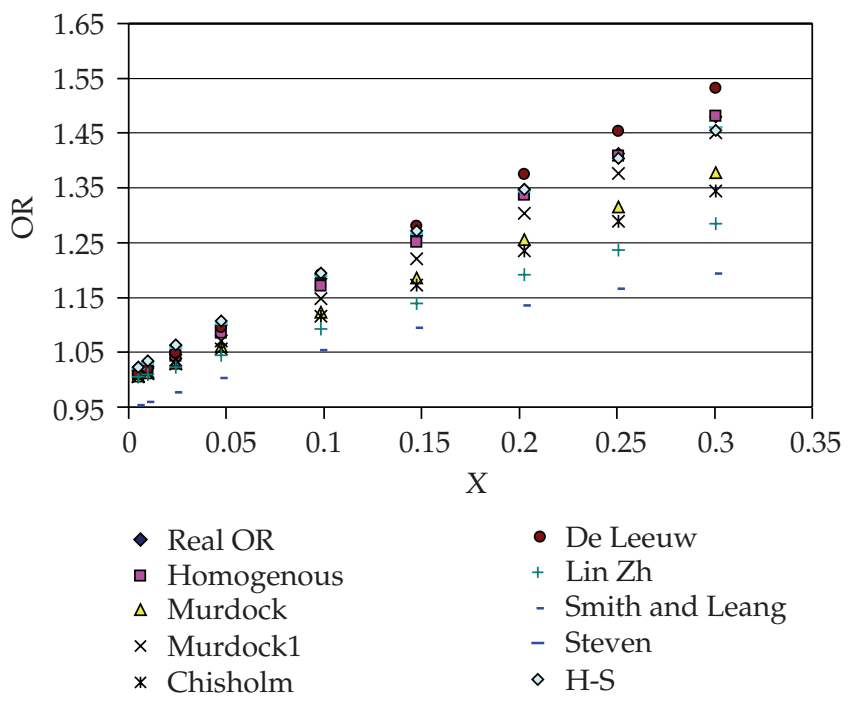

Figure 23: Comparison of models under $\beta=0.75, P=6 \mathrm{MPa}$, and $\mathrm{Fr}_{g}=3.5$.

\subsection{Comparison of H-S OR Model and the Eight Previous OR Models}

Compare new model to 8 old models with the condition of pressure $P$ varied from 0.15 to 6.0 MPa, beta ratio varied from 0.4 to 0.75 , gas densimetric Froude number $\mathrm{Fr}_{g}$ varied from 0.5 to 5.5 , the modified Lockhart-Martinelli parameter $X$ varied from 0.002 to 0.3 , the ratio of the gas to total mass flow rate $x$ varied from 0.5 to 0.99 . The data used for comparison is independent data different from training data. A Part of independent data was obtained from 


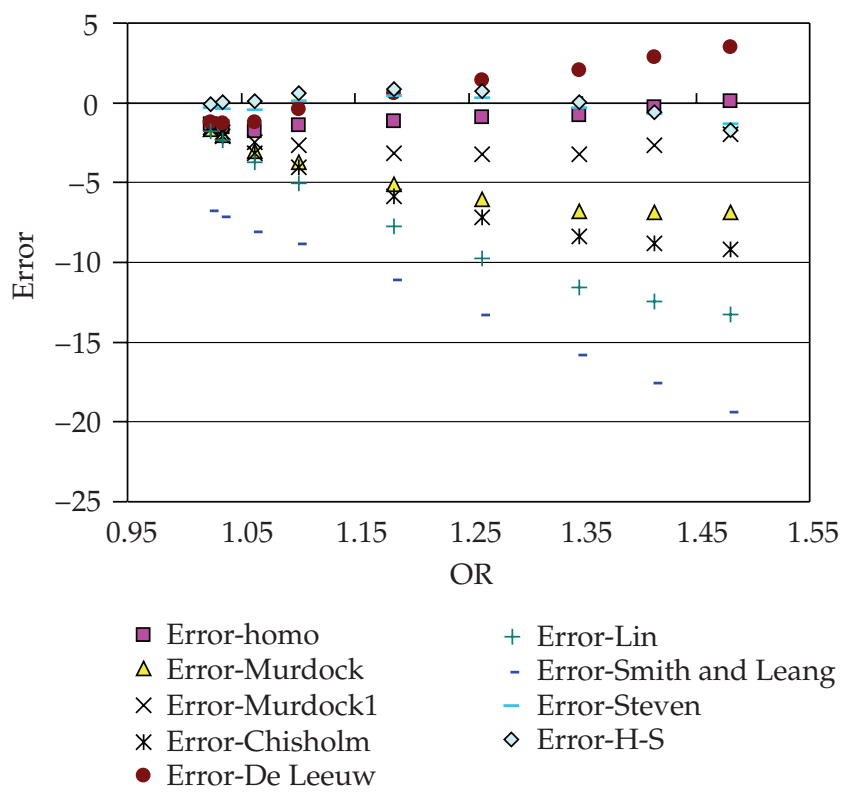

Figure 24: Comparison errors of models under $\beta=0.75, P=6 \mathrm{MPa}$, and $\mathrm{Fr}_{g}=3.5$.

NEL's report. Figures 13, 14, 15, 16, 17, 18, 19, 20, 21, 22, 23, and 24 are a part of the compared results.

These figures show that H-S model can accurately predict Venturi OR in all kinds of flow conditions, the error of H-S wet gas model is stable with OR increasing, and within $5 \%$. It again proved that new wet gas model has good adaptability and wide application range. Particularly, as the wet gas flow fluctuate intensively under low pressure, all old OR models cannot predict OR accurately, the absolute of maximum error almost reached $40 \%$. However, the new wet gas model reflects this change perfectly, the prediction OR has the same distribution with real OR. This is mainly because the homogenous model can well reflect the fluctuation of real OR, and the H-S model has inherited this ability. NEL' data have evidence trends because it is obtained in middle and high pressure. Even though, old correlations predicted errors are also large than $\mathrm{H}-\mathrm{S}$ correlation, they varied from $10 \%$ to $-35 \%$.

\section{Conclusions}

Separation and homogeneous assumptions reflect the wet gas flow character, so a correlation combining these two assumptions performed well than each single one. The H-S model has inherited merits of homogeneous correlation and separation correlation, and can predict Venturi over reading accurately with the conditions of pressure varied from 0.15 to $6 \mathrm{MPa}$, beta ratio varied from 0.4 to 0.75 , gas densimetric Froude number varied from 1 to 5.5, the modified Lockhart-Martinelli parameter varied from 0.002 to 0.3 , the ratio of the gas to total mass flow rate varied from 0.5 to 0.99 . The prediction error of $\mathrm{H}-\mathrm{S}$ model is within $\pm 6.5 \%$.

\section{Acknowledgments}

This work was supported by the High-Tech Research and Development Program of China, numbers 2006AA04Z167, 2007AA04Z180, and supported in part by the National Natural 
Science Foundation of China under the project Grant no. 60573125. The author would like to thank National Engineering Laboratory for providing the reports and professional advice on the web.

\section{References}

[1] J. Agar and D. Farchy, "Wet gas metering using dissimilar flow sensors: theory and field trial results," in Proceedings of the SPE Annual Technical Conference and Exhibition, pp. 1-6, San Antonio, Tex, USA, September-October 2002, SPE 77349.

[2] A. Lupeau, B. Platet, P. Gajan, A. Strzelecki, J. Escande, and J. P. Couput, "Influence of the presence of an upstream annular liquid film on the wet gas flow measured by a Venturi in a downward vertical configuration," Flow Measurement and Instrumentation, vol. 18, no. 1, pp. 1-11, 2007.

[3] Y. Geng, J. Zheng, and T. Shi, "Study on the metering characteristics of a slotted orifice for wet gas flow," Flow Measurement and Instrumentation, vol. 17, no. 2, pp. 123-128, 2006.

[4] J. W. Murdock, "Two-phase flow measurements with orifices," Journal of Basic Engineering, vol. 84, no. 4, pp. 419-433, 1962.

[5] R. V. Smith and J. T. Leang, "Evaluations of correlations for two-phase, flowmeters three current-one new," Journal of Engineering for Power, vol. 97, no. 4, pp. 589-593, 1975.

[6] D. Chisholm, "Research note: two-phase flow through sharp-edged orifices," Journal of Mechanical Engineering Science, vol. 19, no. 3, pp. 128-130, 1977.

[7] Z. H. Lin, "Two-phase flow measurements with sharp-edged orifices," International Journal of Multiphase Flow, vol. 8, no. 6, pp. 683-693, 1982.

[8] G. Toma, "Practical test-functions generated by computer algorithms," in Proceedings of the International Conference on Computational Science and Its Applications (ICCSA '05), vol. 3482 of Lecture Notes in Computer Science, pp. 576-584, Singapore, May 2005.

[9] R. de Leeuw, "Wet gas flow measurement using a combination of Venturi meter and a tracer technique," in Proceedings of the 12th North Sea Flow Measurement Workshop, Peebles, Scotland, October 1994.

[10] R. N. Steven, "Wet gas metering with a horizontally mounted Venturi meter," Flow Measurement and Instrumentation, vol. 12, no. 5-6, pp. 361-372, 2001.

[11] NEL, "Effects of two-phase flow on single phase flow meters," Flow Measurement Guidance Note, no. 3, pp. 1-3, July 1997.

[12] NEL, The Evaluation of Dry Gas Meters in Wet Gas Condition, NEL, London, UK, 2002.

[13] NEL, The Evaluation of Wet Gas Metering Technologies for Offshore Application: Part1-Differential Pressure Meters, Flow Measurement Guidance Note, no. 40, NEL, London, UK, Feb 2003.

[14] C. Britton, W. Seidl, and J. Kinney, "Experimental, wet gas data for a Herschel style Venturi," in Proceedings of the 5th International Symposium on Fluid Flow Measurement, pp. 1-8, Arlington, Va, USA, April 2002.

[15] T. Kegel, "Wet gas measurement," in Proceedings of the 4th CIATEQ Seminar on Advanced Flow Measurement, pp. 1-7, Boca del Rio, Mexico, July 2003.

[16] F. Lide, Z. Tao, and J. Ningde, "A comparison of correlations used for Venturi wet gas metering in oil and gas industry," Journal of Petroleum Science and Engineering, vol. 57, no. 3-4, pp. 247-256, 2007.

[17] G. F. Hewitt, Measurement of Two Phase Flow Parameter, Academic Press, New York, NY, USA, 1978.

[18] G. B. Wallis, One-Dimensional Two-Phase Flow, McGraw-Hill, New York, NY, USA, 1969.

[19] C. Zhihang, C. Bolin, and Z. Zaisan, Gas-Liquid Two Phase Flow and Heat Transfer, Mechanical Industry Press, Peking, China, 1983.

[20] L. Zonghu, W. Shuzhong, and W. Dong, Gas-Liquid Two Phase Flow and Bioling Heat Transfer, Xi'An Jiao Tong University Press, Xi'An, China, 2003.

[21] L. Yi, The study of wet gas measurement technology for applications: Venturi tube, Master's Degree Dissertation, Tianjin University, Tianjin, China, 2005.

[22] ISO 5167-3:2003, "Measurement of fluid flow by means of pressure differential devices inserted in circular cross-section conduits running full-part 3: nozzles and Venturi nozzles".

[23] ISO 5167-1:2003, "Measurement of fluid flow by means of pressure differential devices inserted in circular cross-section conduits running full-part 1: general principles and requirements".

[24] ISO 5167-4:2003, "Measurement of fluid flow by means of pressure differential devices inserted in circular cross-section conduits running full-part 4: Venturi tubes". 


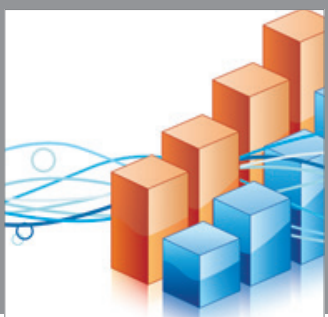

Advances in

Operations Research

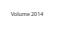

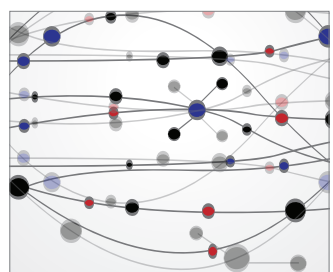

\section{The Scientific} World Journal
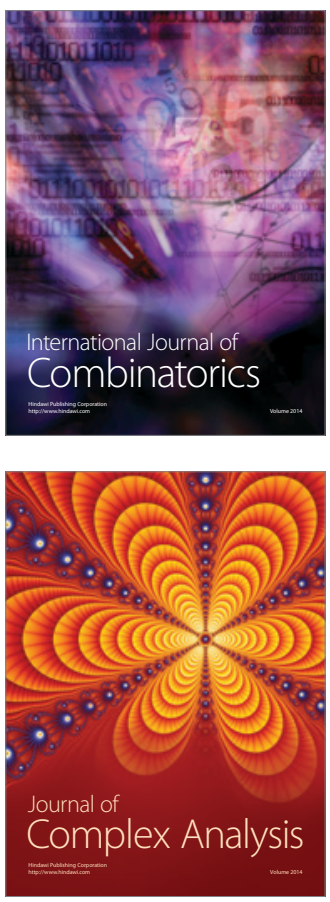

International Journal of

Mathematics and

Mathematical

Sciences
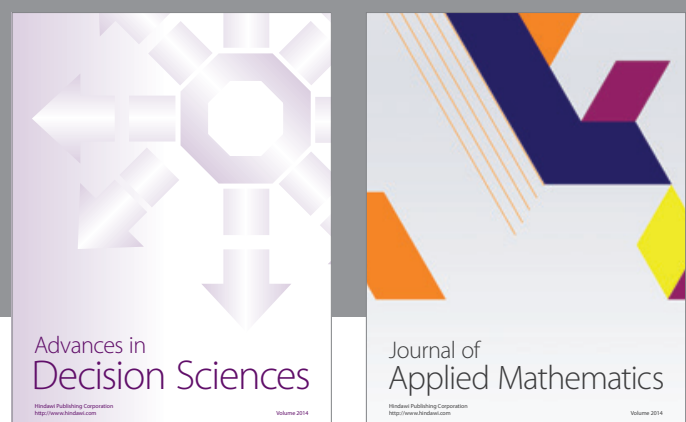

Journal of

Applied Mathematics
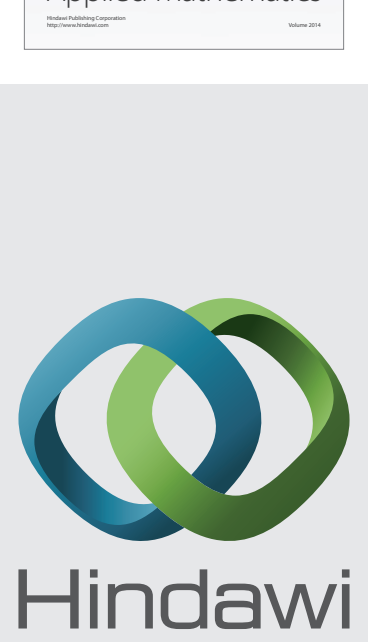

Submit your manuscripts at http://www.hindawi.com
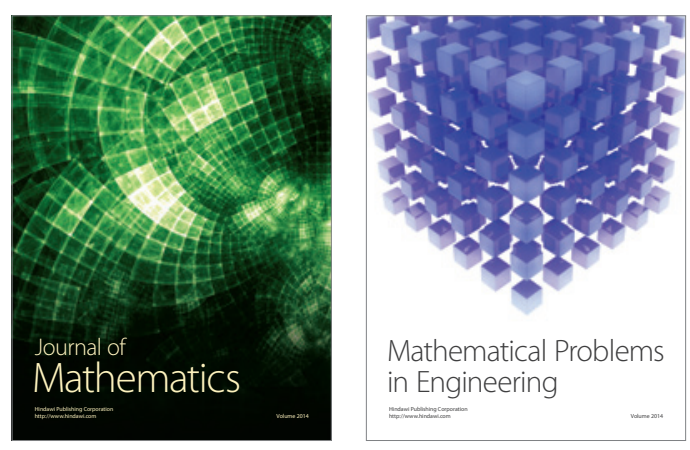

Mathematical Problems in Engineering
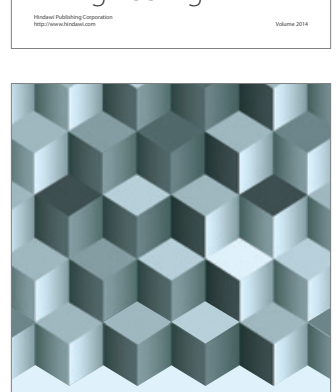

Journal of

Function Spaces
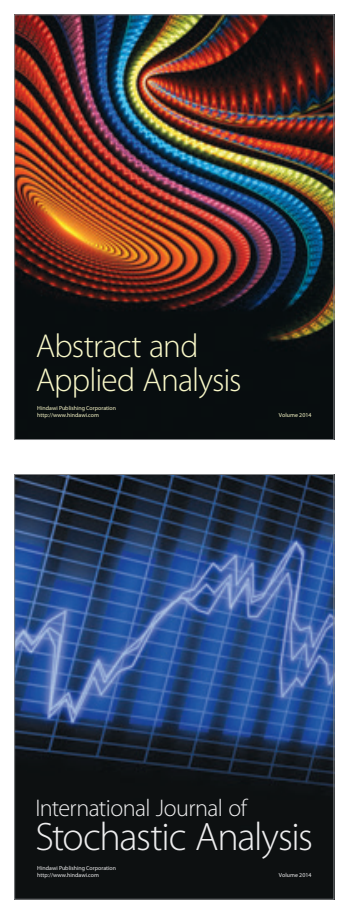

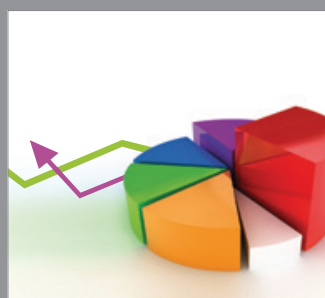

ournal of

Probability and Statistics

Promensencen
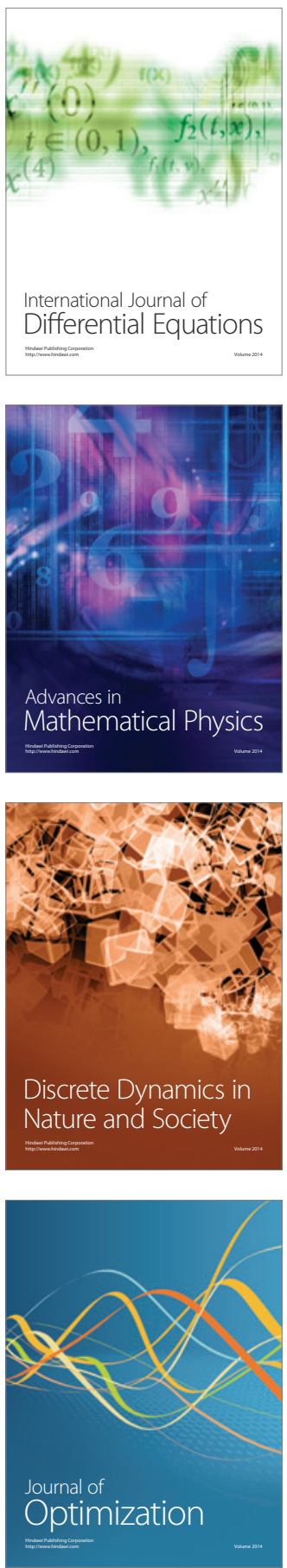